\title{
Force-induced structural transitions in cross-linked DNA films
}

\author{
A. André · F. Fontaine-Vive $\cdot$ H. M. Möller · T. Fischer · \\ G. Maret $\cdot$ V. T. Forsyth $\cdot$ T. Gisler
}

\begin{abstract}
We report on the preparation and characterization of wet-spun films of sodium DNA in which intermolecular cross-links were introduced following formaldehyde treatment. Raman scattering shows that the DNA in moderately cross-linked films is mainly in the B conformation. Stretching experiments show a transition from plastic to elastomeric behavior with increasing exposure to the cross-linking agent. Elastomeric DNA films are strongly disordered. X-ray diffraction shows that stretching of moderately cross-linked films under controlled high humidity conditions results in increased molecular orientation as well as the appearance of meridional reflections at 7.4 7.8 and $8.2 \AA$. These reflections are not observed for any of the classical conformations associated with mixed sequence DNA, and may arise from extended base-pair stacking in a stretched DNA structure.
\end{abstract}

A. André · T. Fischer · G. Maret · T. Gisler $(\bowtie)$

Universität Konstanz, Fachbereich Physik,

78457 Konstanz, Germany

e mail: thomas.gisler@uni konstanz.de

\section{A. André}

Université Louis Pasteur, 4 rue Blaise Pascal,

67070 Strasbourg, France

F. Fontaine Vive $\cdot$ V. T. Forsyth

Institut Laue Langevin, 6 rue Jules Horowitz BP 156,

38042 Grenoble, France

H. M. Möller

Universität Konstanz, Fachbereich Chemie,

78457 Konstanz, Germany

V. T. Forsyth

EPSAM/ISTM Research Institutes, Keele University,

Staffordshire ST5 5BG, UK
Keywords DNA structure - DNA cross-linking · X-ray fibre diffraction · Raman scattering

\section{Introduction}

Within the last years single-molecule experiments have provided unprecedented insights into the micromechanics of DNA (Lavery et al. 2002). For end-to-end distances smaller than the relaxed contour length $\left(l_{0}=16.5 \mu \mathrm{m}\right.$ for $\lambda$-DNA with $4.85 \times 10^{4}$ base pairs), the force-extension curve of double-stranded DNA is accurately described by the wormlike-chain model for semiflexible polymers (Smith et al. 1992). At higher forces of about $50110 \mathrm{pN} \mathrm{a}$ completely new behavior is observed: the force-extension curve becomes flat, and the molecule can be overstretched at almost constant force up to an extension of $1.7 l_{0}$ (Smith et al. 1996; Cluzel et al. 1996). This transition to an overstretched state is reversible, and the exact force for overstretching depends on ionic strength (Williams et al. 2001; Williams et al. 2001), the presence of gaps (nicks) in the sugar-phosphate backbone (Sarkar et al. 2001), and on the relative contents of dG-dC and dA-dT pairs (Rief et al. 1999).

The origin of the plateau in the force-extension curve is still under debate: based on the $\mathrm{pH}$ and ionic strength dependence of the single-molecule force-extension curves, Rouzina and Bloomfield proposed force-induced melting of double-stranded DNA as its origin (Rouzina and Bloomfield 2001; Rouzina and Bloomfield 2001). The assumption of a first-order transition between two structures separated by an energy barrier which is at the basis of this denaturation scenario was also adopted by the authors who interpret the plateau in the force-extension curve in terms of a coexistence between B-DNA and an 
underwound duplex structure with intact base pairing (Cluzel et al. 1996; Marko 1998; Ahsan et al. 1998; Sarkar et al. 2001; Storm and Nelson 2003; Lai and Zhou 2003). An intermediate scenario for the plateau in the force-extension curve at low salt concentration is a transition to a structure with denatured segments nucleating at gaps in the sugar-phosphate backbone (Smith et al. 1996; Cocco et al. 2004).

Recent atomistic molecular dynamics (MD) simulations on short DNA including explicit solvent have suggested that locally denatured DNA conformations are responsible for the force plateau (Harris et al. 2005; Piana 2005). The simulations by Piana (Piana 2005) indicate that the structural transition associated with the force plateau is continuous rather than first-order. The DNA structures predicted by these simulations are at odds with results of earlier MD simulations (MacKerell and Lee 1999; Kosikov et al. 1999) and molecular modelling (Lebrun and Lavery 1996) which suggest that high forces induce the formation of double-stranded structures which are, depending on the pulling pathway and the base pair composition, doublehelical with increased pitch, or non-helical with strongly inclined base pairs. This, in turn, is supported by birefringence measurements (Wilkins et al. 1951) and X-ray diffraction data on elongated drying DNA fibres (Greenall et al. 2001) which followed up early data by Wilkins suggesting an increase in the base pair spacing from 3.4 to about $5.6 \AA$ in stretched oriented fibres (Wilkins 1961). These data are, however, not readily compared to the data from single molecules since mechanically stretched DNA fibres and films show plastic flow and lack (even at a high degree of orientation) the characteristic force plateau observed in single-molecule experiments (Rupprecht 1970).

In this publication, we report on the preparation and characterization of wet-spun DNA films in which plastic flow was suppressed by intermolecular cross-linking with formaldehyde. Gel electrophoresis, swelling experiments, and mechanical stretching experiments have been carried out in combination with Raman spectroscopy and X-ray diffraction in order to probe the macroscopic elasticity of the films and the molecular changes in the DNA associated with film stretching at controlled humidity. Cross-linked DNA films show a transition to elastomeric behavior with increasing exposure to the cross-linking agent. X-ray diffraction shows that the mechanical stretching of crosslinked films enhances the crystalline order and leads to meridional reflections corresponding to periodicities of 7.4 7.8 and 8.2 ̊. Similar features are found in unstretched untreated films prepared by spinning at high speed. High cross-link densities, on the other hand, induce strong disorder in the films.

\section{Materials and methods}

Preparation and cross-linking of DNA films

Oriented DNA films were prepared by wet-spinning aqueous solutions of DNA from salmon testes (Fluka) or from calf thymus (Sigma) at concentrations of 1.58 and $1.37 \mathrm{~g} / \mathrm{l}$, respectively, in $0.15 \mathrm{M}$ sodium chloride, $3 \mathrm{mM}$ sodium citrate tribasic dihydrate (Sigma) and $0.05 \mathrm{mM}$ disodium EDTA (Sigma) (Rupprecht 1963; Rupprecht 1966) at $\mathrm{pH} \approx 7$. A $75 \%$ (by volume) aqueous solution of ethanol with $0.2 \mathrm{M} \mathrm{NaCl}$ served as spinning bath. Typically, the films consist of 2040 layers of DNA fibres each of thickness $2050 \mu \mathrm{m}$. After bathing in $75 \%$ ethanol containing $0.03 \mathrm{M}$ $\mathrm{NaCl}$ at $5^{\circ} \mathrm{C}$ overnight, the films were dried in a desiccator for one day at the same temperature. After storage at $75 \%$ relative humidity (r. h.) at room temperature for at least 3 days, the films were removed from the spinning cylinders and transferred to a closed box at room temperature and $98 \%$ r. h. X-ray diffraction showed that the transfer to high humidity induced a transition from A- to semicrystalline BDNA. Films were cross-linked in formaldehyde vapour over a hot plate, or, alternatively, by brushing with aqueous formaldehyde solution at room temperature.

\section{Characterization of cross-linking}

Solutions of marker DNA (N 3234 L, New England Biolabs, $c=300 \mu \mathrm{g} / \mathrm{ml} ; 50,150,300,500$, and 766 base pairs) and of linearized $\lambda$-DNA (New England Biolabs; 48,502 base pairs) in saline phosphate buffer (PBS) were mixed with equal volumes of formaldehyde solution (37\%, Riedel-de Haën), whose $\mathrm{pH}$ had been adjusted with sodium hydroxide and hydrochloric acid, respectively. $\lambda$-DNA was endlabelled with biotinylated and thiol-marked oligomers (ThermoElectron) in order to avoid ring formation (Lehner et al. 2006). DNA solutions were mixed with equal volumes of formaldehyde solution and incubated overnight at room temperature. Gel electrophoresis in $1 \%$ agarose was performed on samples in PBS from which the formaldehyde had been removed by precipitation in ethanol. Bands were visualized by ethidium bromide staining, and molecular weights of cross-linking products were determined by calibrating with the marker bands using the method of Southern (Southern 1979). Cross-linking of DNA in wet-spun films was characterized by swelling the films in deionized water.

\section{Raman scattering}

Raman spectra were measured with a grating microspectrometer (LabRAM HR 800, HORIBA Jobin Yvon), using 
a frequency-doubled Nd:YAG laser at $532 \mathrm{~nm}$ for excitation and a cooled CCD detector. Typically, $10 \mathrm{~mW}$ were focussed to a spot about $4 \mu \mathrm{m}$ in diameter. Ten spectra with an exposure time of $60 \mathrm{~s}$ each were averaged. Two notch filters with a bandwidth of about $200 \mathrm{~cm}^{-1}$ were used before the CCD to reject elastically scattered light. The instrumental resolution was about $2.5 \mathrm{~cm}^{-1}$. DNA films were stretched in a hermetically sealed sample cell in which the relative humidity was set to $98 \%$ by equilibration with a saturated $\mathrm{K}_{2} \mathrm{SO}_{4}$ solution.

\section{X-ray diffraction}

X-ray diffraction was performed using a rotating anode source (Schneider) operated at $40 \mathrm{kV}$ and $100 \mathrm{~mA}$ at a wavelength of $1.54 \AA$. Diffraction patterns were recorded using an image plate with a diameter of $345 \mathrm{~mm}$ (MAR 345 , mar research). DNA films were mounted in a sample cell which allows for extension by a linear motor (Newport) and simultaneous measurement of the force using a sensor with a range of $10 \mathrm{~N}$ (31E, Althen). The humidity inside the sample cell was adjusted with a saturated $\mathrm{K}_{2} \mathrm{SO}_{4}$ solution to $98 \% \mathrm{r}$. h. and monitored with a hygrometer. The sample cell was hermetically sealed with thin polyimide or PET windows. In order to enhance the intensity of the $3.4 \AA$ base pair reflection the film axis (which was also the stretching axis) was tilted away from the plane perpendicular to the direction of the incoming X-ray beam.

\section{Results}

\section{Characterization of cross-linking}

DNA films cross-linked for more than $10 \mathrm{~min}$ in formaldehyde vapour exhibit swelling by several times the original volume in deionized water, but cannot be dissolved, showing the presence of a high-molecular weight network. DNA films that are not cross-linked, on the other hand, dissolve completely in deionized water within less than 2 min Gel electrophoresis on marker DNA shows that the treatment with formaldehyde results in a shift of the bands towards larger molecular weights (see Fig. 1) when the $\mathrm{pH}$ of the formaldehyde is between 9 and 10, showing that intermolecular cross-linking has taken place. In particular, the shortest fraction with 50 base pairs is strongly depleted in the cross-linked samples. The reduced intensity of the bands in the cross-linked sample is probably due to losses incurred by the ethanol precipitation. However, bands at higher mobilities indicating formation of compact molecules by intramolecular cross-links cannot be detec-

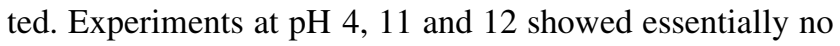

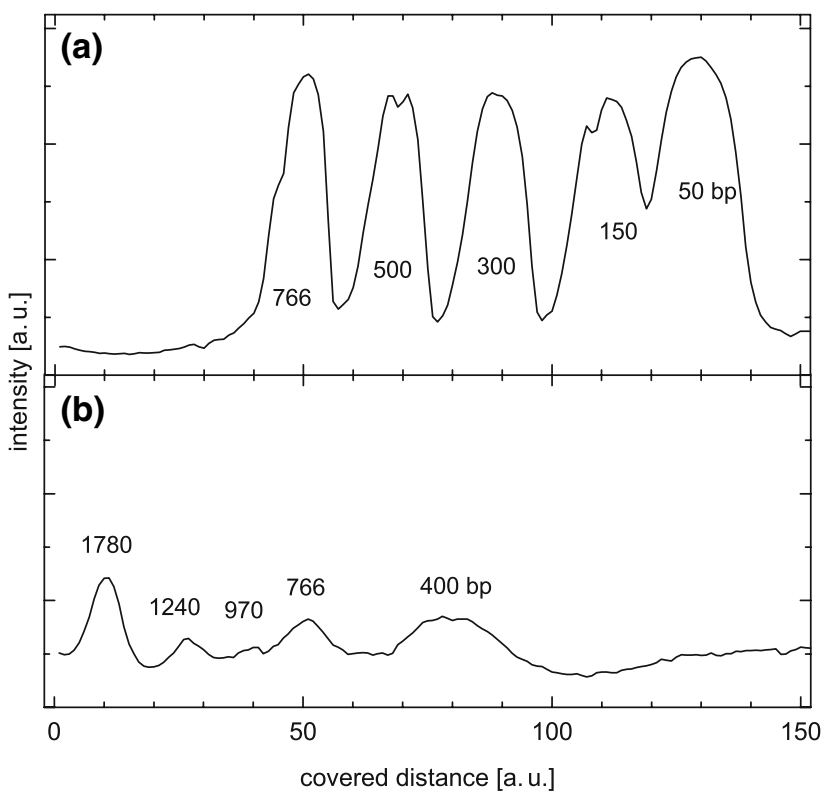

Fig. 1 Agarose gel electrophoresis of (a) untreated marker DNA and (b) marker DNA cross linked in formaldehyde solution at $\mathrm{pH}$ 9. The numbers denote the molecular weight $M$ in units of base pairs

discernible bands. As the electrophilic behavior of formaldehyde and the nucleophilic behavior of amino groups depend on $\mathrm{pH}$, we expect an optimal cross-linking that appears, according to the experimental result, around pH 910.

Formaldehyde treatment of $\lambda$-DNA end-labeled with biotin and thiol shows a strong depletion of the $\lambda$-DNA band (data not shown) and, at $\mathrm{pH}$ between 9 and 10, a broad unresolved signal at lower mobilities due to the formation of a wide distribution of high molecular weights by intermolecular cross-links. At $\mathrm{pH} 4$ no cross-linking was observed, while at $\mathrm{pH} 11$ and 12 the cross-linking is so strong that most of the signal is localized close to the origin. High-molecular weight products do not penetrate into the gel and remain at the starting point, in line with the strong swelling of cross-linked films.

\section{Structure of cross-linked DNA films}

\section{Moderately cross-linked films}

X-ray diffraction patterns from DNA films cross-linked in formaldehyde vapour at about $80^{\circ} \mathrm{C}$ for 10 min show the characteristic features of B-DNA, namely the strong meridional reflection from the base pair stacking distance $3.4 \AA$, and off-meridional diffraction reflecting the helical symmetry (Franklin and Gosling 1953; Saenger 1988) (data not shown). Exposing the films to formaldehyde vapour at about $50^{\circ} \mathrm{C}$ for $40 \mathrm{~min}$ allows a higher degree of 

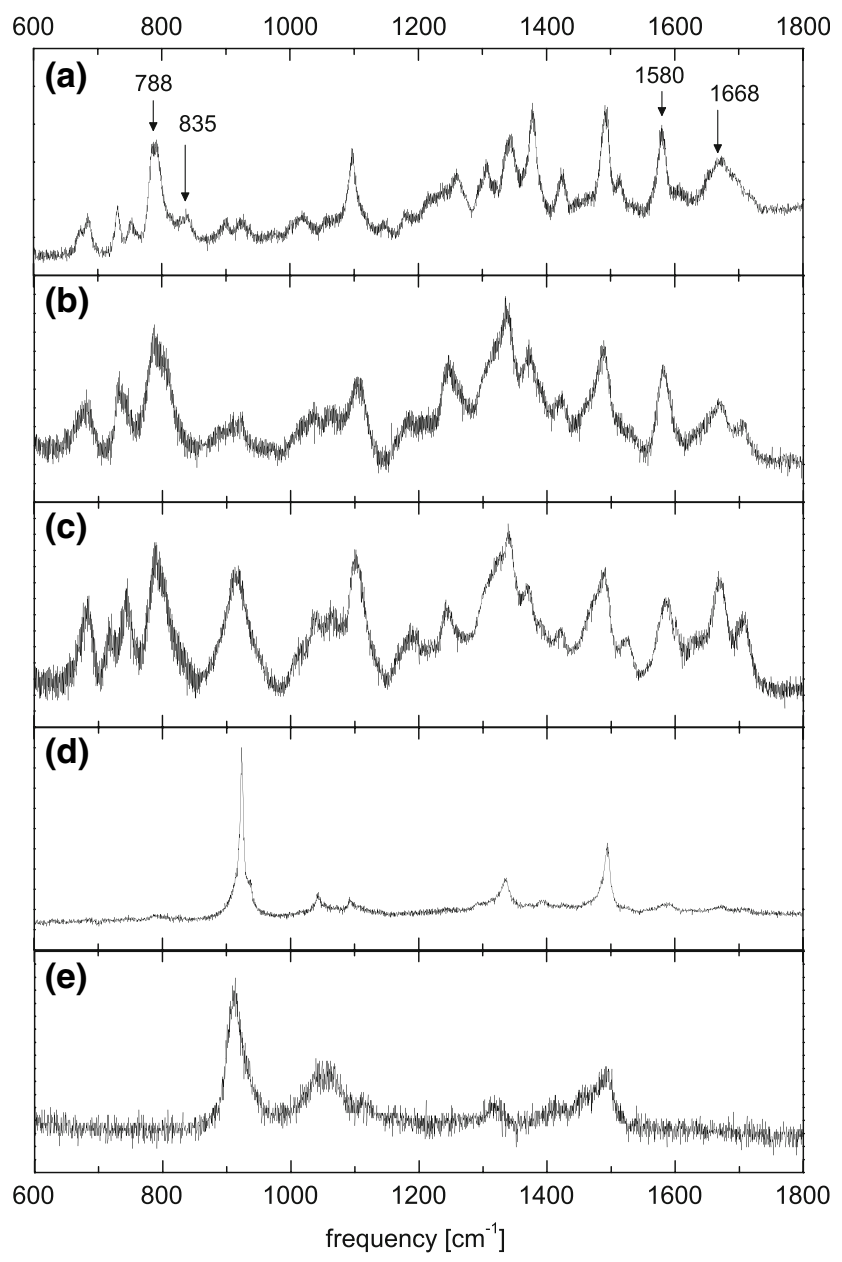

Fig. 2 Raman spectra of (a) an untreated NaDNA film at 98\% r. h.; (b) film treated with formaldehyde solution; (c, d) films exposed to formaldehyde vapour for 10 and $30 \mathrm{~min}$, respectively. e Raman spectrum of pure formalin. The strong bands in (c) and (d) at 923 and $1,495 \mathrm{~cm}^{-1}$ are probably due to paraformaldehyde. Spectra (b) and (c) are background corrected data

cross-linking to be attained, which results in a slightly enhanced intensity of the $3.4 \AA$ reflection.

Cross-linking in formaldehyde vapour induces characteristic changes to the Raman spectra of B-DNA (Fig. 2a): the purine and pyrimidine ring vibration bands at 788 and at $1,580 \mathrm{~cm}^{-1}$ (Peticolas et al. 1987) are broadened, but unchanged in frequency, indicating that the nucleobases remain intact under the formaldehyde treatment. The enhanced intensity of the $1,343 \mathrm{~cm}^{-1}$ band is accompanied by a weakening of the one at $1,378 \mathrm{~cm}^{-1}$. In addition, the antisymmetric phosphodiester stretch vibration band at $835 \mathrm{~cm}^{-1}$ in B-DNA becomes very weak when the films are exposed to formaldehyde solution or vapour for relatively short times (see Fig. 2b, c). This vibration is a marker for B-DNA as it appears when the deoxyribose ring is in the $\mathrm{C}_{2}$--endo conformation (Peticolas et al. 1987). The $\mathrm{PO}_{2}^{-}$symmetric stretch vibration band at $1,096 \mathrm{~cm}^{-1}$ is largely unchanged for moderate cross-link densities suggesting that the phosphate backbone is not involved in cross-linking. The thymine carbonyl stretch vibration band at $1,668 \mathrm{~cm}^{-1}$ is split in cross-linked films, while the intensity is not substantially changed. Bands characteristic of A-DNA (such as the one at $807 \mathrm{~cm}^{-1}$ ) are absent.

\section{Strongly cross-linked films}

When the films are exposed to formaldehyde vapour for more than about $30 \mathrm{~min}$, the nucleobase ring vibration bands at 788 and $1,580 \mathrm{~cm}^{-1}$ disappear behind a strong background probably due to luminescence from salt ions (see Fig. 2d). Washing the sample did not change this result. The Raman spectra of such strongly cross-linked films then resemble the spectrum of aqueous formaldehyde solution (formalin), with prominent bands at 923 and $1,495 \mathrm{~cm}^{-1}$ probably due to paraformaldehyde. The Raman spectrum of formalin shows peaks at 907, 1,041 and $1,492 \mathrm{~cm}^{-1}$ (see Fig. 2e), in agreement with literature (Huang et al. 2003). Strongly cross-linked films are heavily disordered; their X-ray diffraction patterns exhibit only a low-angle halo.

\section{Elasticity and structure of stretched DNA films}

\section{Untreated films}

Upon stretching along the fibre axis, untreated NaDNA films show necking and a rapid increase of the force already at very small strains, followed by a plateau which extends up to strains $\epsilon \approx 3$, and a significant hysteresis which decreases in subsequent stretch-release cycles (see Fig. 3a). Such films can be stretched to about $300 \%$ of their original length before they break. We observe that weak stretching noticeably enhances the intensity of the B-DNA diffraction spots and reduces the continuous scattering between the diffraction spots which is prominent in untreated films at high humidity.

\section{Moderately cross-linked films}

Films moderately cross-linked in formaldehyde vapour for about 10 min show both the $3.4 \AA$ meridional reflections associated with base pair stacking, and the cross-like off-meridional diffraction characteristic for semi-crystalline B-DNA (see Fig. 4). We also observe a meridional reflection corresponding to an axial periodicity of $4.2 \AA$. A reflection with the same periodicity was noted in earlier fibre diffraction studies on the double stranded alternating DNA 

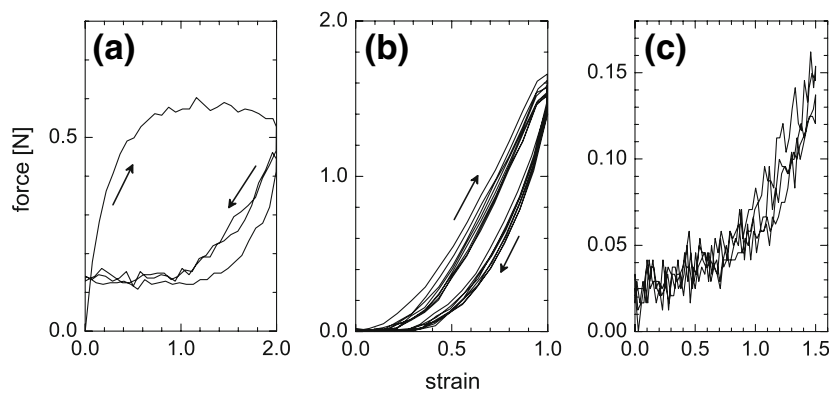

Fig. 3 Force strain curves of (a) an untreated DNA film and (b, c) DNA films cross linked in formaldehyde vapour for about $40 \mathrm{~min}$ at $50^{\circ} \mathrm{C}$ and $60 \mathrm{~min}$ at $80^{\circ} \mathrm{C}$, respectively. The relative humidity (over $92 \%$ ) was controlled by humid nitrogen flowing through the sample cell. Differences in the absolute force scales in a c are due to different film thicknesses

copolymer poly $[\mathrm{d}(\mathrm{GC})] \cdot \operatorname{poly}[\mathrm{d}(\mathrm{GC})]$ in the $\mathrm{B}$ conformation (Arnott et al. 1980). Furthermore, a weak meridional reflection corresponding to an axial periodicity of about $7.8 \AA$ is observed. This reflection has not been reported so far.

Films cross-linked in formaldehyde vapour at $50^{\circ} \mathrm{C}$ for 40 min can be stretched relatively reversibly to a strain $\epsilon \approx 1$ with a pronounced hysteresis (see Fig. 3b). Under stretching, we observe in addition to the reflections characteristic for B-DNA which are more intense than the ones for samples cross-linked at $80^{\circ} \mathrm{C}$ a meridional reflection corresponding to an axial periodicity of $7.4 \AA$ (see Fig. 5).

The X-ray diffraction pattern of a stretched film that has been treated with formaldehyde by brushing shows reduced intensity on the first three layer lines of the B-DNA diffraction pattern (see Fig. 6). Beyond this, the data shown in Fig. 6 show a set of sharp diffraction features that clearly

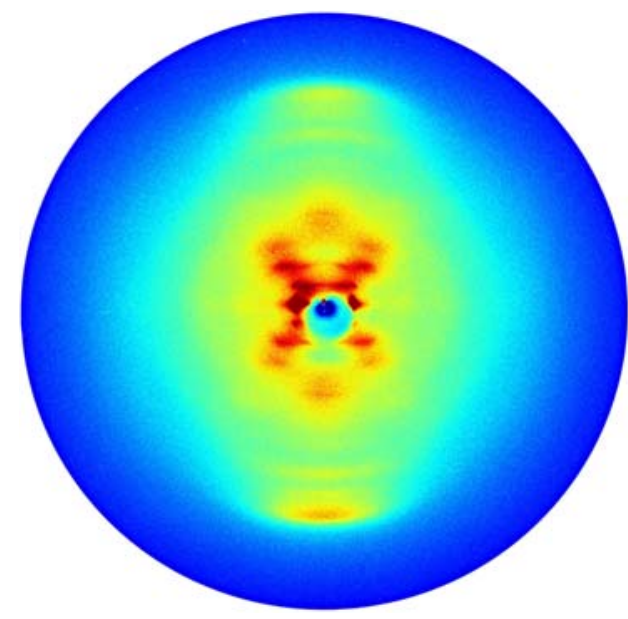

Fig. 4 Left $\mathrm{X}$ ray diffraction pattern of a NaDNA film from salmon testes, cross linked in formaldehyde vapour for $10 \mathrm{~min}$, stretched at $98 \%$ r. h. to a strain $\epsilon \quad 0.05$. Right Assignment of reflections. Dotted line equatorial axis; dash dotted line meridional axis. Blue dashes $\mathrm{B}$ DNA reflections. Red dashes stretching induced reflections. Sample arise from a quite different structure. These features are consistent with an axial periodicity of $31 \AA$ which is substantially shorter than that of B-DNA ( $34 \AA$ ), and an intermolecular separation of about $20 \AA$. As shown in the schematic diagram in Fig. 6, the intense second layer line reflection from this set of features corresponds to an axial periodicity of about $14.3 \AA$. In addition, we observe meridional reflections corresponding to periodicities of about 7.6 and $8.2 \AA$. Of these two meridional reflections, the one at $7.6 \AA$ is also visible in the diffraction pattern for the unstretched film.

\section{Strongly cross-linked films}

Strongly cross-linked films (exposed to formaldehyde vapour for more than $60 \mathrm{~min}$ ) can be stretched completely reversibly already in the first stretch-release cycle up to maximal strains $\epsilon \approx 1.6$ (see Fig. 3c). Strongly crosslinked films which are kept at a fixed strain $\epsilon=1.7$ show no measurable reduction of the force over more than $3 \mathrm{~h}$. Stretching of strongly cross-linked films does not significantly enhance the sample order, as seen by the recorded diffraction patterns (data not shown).

\section{Discussion}

Gel electrophoresis shows that treatment with formaldehyde leads to intermolecular cross-linking. While the formation of covalent intramolecular cross-links by formaldehyde within individual DNA chains has been extensively studied (Collins and Guild 1968; Freifelder and

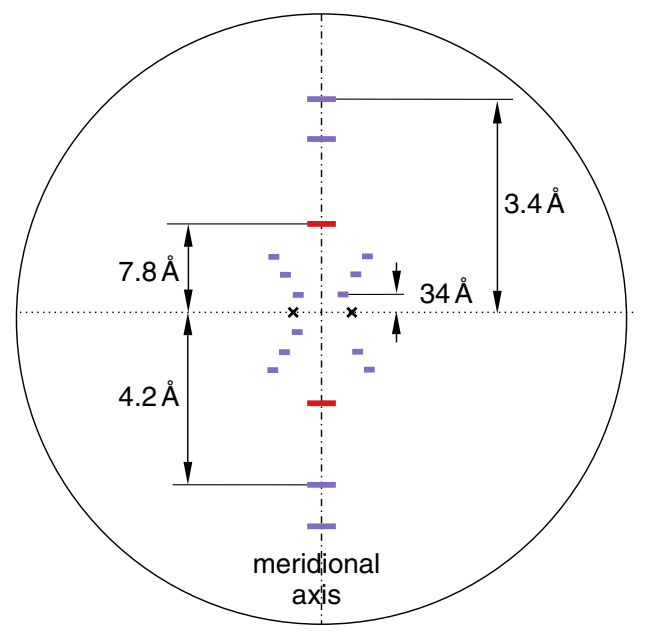

detector distance: $250 \mathrm{~mm}$. The meridional axis coincides with the stretching direction. The circle marks the resolution $2.6 \AA$. Note the stretching induced broad meridional reflection corresponding to the axial periodicity of $7.8 \AA$ 


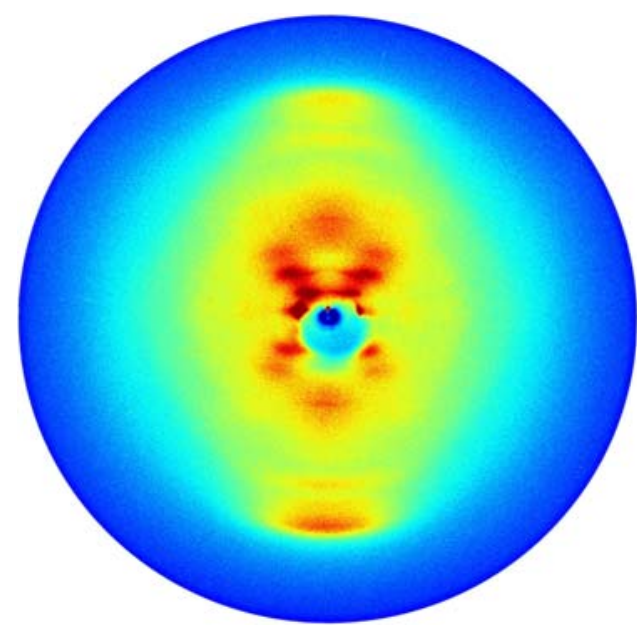

Fig. 5 Left $\mathrm{X}$ ray diffraction pattern of a NaDNA film from salmon testes, cross linked in formaldehyde vapour for $40 \mathrm{~min}$ at $50^{\circ} \mathrm{C}$, stretched at $98 \%$ r. h. to a strain $\epsilon \approx 0.45$. Right Assignment of reflections. Dotted line equatorial axis; dash dotted line meridional axis. Blue dashes B DNA reflections. Red dashes stretching induced

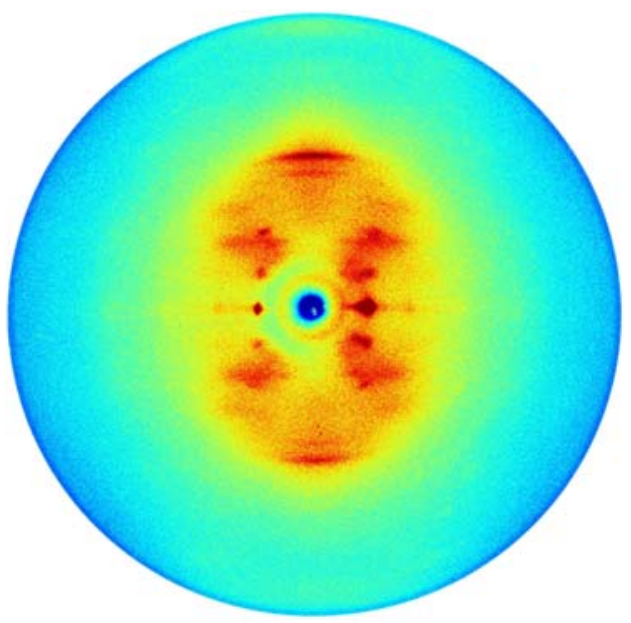

Fig. 6 Left $\mathrm{X}$ ray diffraction pattern of a NaDNA film from salmon testes cross linked by brushing with formaldehyde solution and stretched at $98 \%$ r. h. to a strain $\epsilon \approx 2$. Right Assignment of reflections. Dotted line equatorial axis; dash dotted line meridional axis. Blue dashes B DNA reflections. Red dashes stretching induced reflections. Crosses equatorial reflections corresponding to the intermolecular spacing $20 \AA$. Red circles off meridional reflections

Davison 1963; Beland et al. 1984; Chaw et al. 1980; Huang et al. 1992; Huang and Hopkins 1993), intermolecular cross-links induced by this compound have, to our knowledge, not been reported before. The presence of high-molecular weight networks is also evident from the swelling of cross-linked films.

The Raman spectra from DNA films show that the nucleobase ring vibrations are affected by cross-linking, consistent with the scenario of formaldehyde-induced formation of methylene bridges between the nucleobases

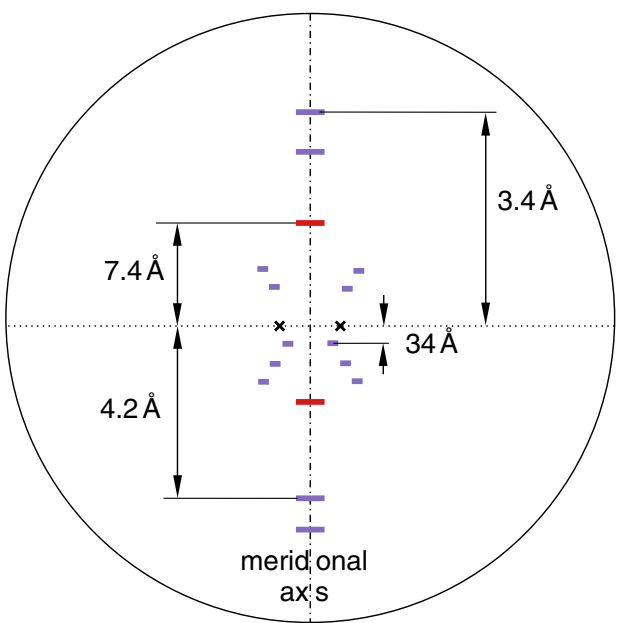

reflections. Crosses equatorial reflections corresponding to the intermolecular spacing $20 \AA$. Sample detector distance: $250 \mathrm{~mm}$. The meridional axis coincides with the stretching direction. Note the broad meridional reflection corresponding to a periodicity of about $7.4 \AA$ A. The circle marks the resolution $2.6 \AA$

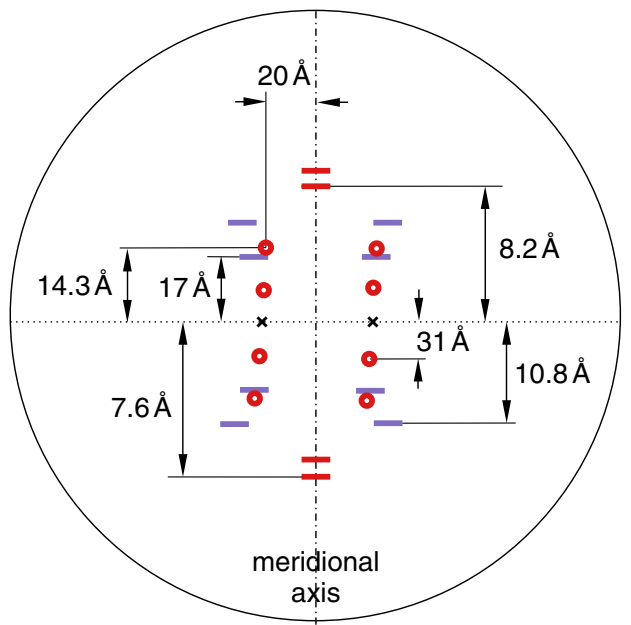

induced by stretching. Note that due to an extended sample detector distance of $400 \mathrm{~mm}$ the base pair reflection from B DNA is not visible. The meridional axis coincides with the direction of stretching. The circle marks the resolution $3.8 \AA$. The $17 \AA$ reflection corre sponds to the second layer line of the B DNA pattern. Note the presence of meridional reflections corresponding to axial periodicities of 7.6 and $8.2 \AA$ which are absent in classical B DNA

(Brodolin 2000). This scenario is also supported by the splitting of the $1,668 \mathrm{~cm}^{-1}$ band (which is associated with the thymine carbonyl stretching vibration) in formaldehyde-treated films. This is clearly different from the effect of thermal denaturation which results in a large intensity increase of the $1,668 \mathrm{~cm}^{-1}$ band (Erfurth and Peticolas 1975). The weakening of the B-DNA marker band at $835 \mathrm{~cm}^{-1}$ upon cross-linking could arise from a changed deoxyribose ring pucker, or, alternatively, from the strong broadening of the neighbouring band at $788 \mathrm{~cm}^{-1}$. Indeed, 
the fact that in some cross-linked samples the band at $835 \mathrm{~cm}^{-1}$ appears as a shoulder, and the presence of characteristic B-DNA X-ray diffraction spots in moderately cross-linked films, support the latter interpretation, implying that moderate cross-linking at high humidity essentially preserves the $\mathrm{B}$ conformation.

The X-ray fibre diffraction data presented here contain many features that have not been noted so far for stretched DNA. In common with earlier studies, there exists no direct way of assigning these features and of reaching an unambiguous model. However some of the observations are thought-provoking. The meridional reflections corresponding to axial periodicities of 7.47 .8 and $8.2 \AA$ may arise from a phase within the sample that has undergone force-induced unwinding of the DNA, resulting in a stretched conformation of the double helix and an extended base pair separation. Unwound structures with an axial distance of $7.4 \AA$ between successive residues were found in molecular modelling of periodic DNA duplexes under mechanical load (Lebrun and Lavery 1996). Axial periodicities of about $7.4 \AA$ have not previously been reported for highly crystalline films or fibres of DNA in any of the classical A, B, or C conformations (Rupprecht and Forslind 1970). Increased axial periodicities have been found in complexes of DNA with bipyridyl-(ethylenediamine) platinum which were interpreted as due to regular intercalation (Lee et al. 2000).

Alternatively, the reflections at 7.4 7.8 $\AA$ might be due to the presence of left-handed Z-DNA (Arnott et al. 1980; Saenger 1988) in stretched films. Such a conformation was postulated by Sarkar et al. in order to explain forceextension curves of undertwisted DNA (Sarkar et al. 2001). A force-induced transition from B- to Z-DNA would require that the torque cannot relax by rotations about gaps in the backbone. While the dense packing of DNA within the film could effectively lead to local torsional constraints even in the presence of a non-negligible density of backbone gaps, the characteristic $43 \AA$ pitch of Z-DNA (Fuller et al. 2004) is not observed in our samples. An increased axial periodicity of about $6.5 \AA$ was also observed by Greenall et al. in stretched NaDNA fibres at low water content (60\% r.h. or lower) (Greenall et al. 2001). At this low relative humidity, the DNA is likely to assume at least partially the A conformation. In contrast, we do not see a meridional diffraction spot at $6.5 \AA$ in untreated films dried to $75 \%$ r. h. in our experiments; in addition, the $7.4 \AA$ reflection which we observe disappears upon drying to $75 \%$ r. h. both in cross-linked and in untreated films. While Greenall et al. observe a clearly reduced intermolecular spacing in stretched drying fibres, our cross-linked samples, whether stretched or not, appear to have intermolecular distances that are equal to or larger than those of classical semicrystalline B-DNA (20 ̊).
Interestingly, a weak meridional diffraction spot corresponding to an axial periodicity of about $7.4 \AA$ was also observed in some cross-linked, unstretched films which suggests that cross-linking alone induces some of the effects that become more pronounced in stretched crosslinked films. Conversely, we also observed meridional $7.4 \AA$ reflections in stretched untreated films, and in very weakly stretched, untreated films produced by spinning at high speed. These unusual X-ray diffraction features in the absence of cross-linkers may arise from the significant internal stresses which develop during the film treatment after spinning. These internal stresses lead to warping in unstretched films.

The meridional reflections at 7.4 and $8.2 \AA$ clearly arise from a periodicity within the DNA molecule. These reflections are observed in DNA from various sources and with different cross-linking and stretching history, indicating that the associated force-induced structural transition is a persistent feature in stretched randomsequence DNA. On the other hand, the variability of the meridional $7.4 \AA$ periodicity over about $0.4 \AA$ suggests that the structural transition induced by force is continuous, which is in agreement with the recent atomistic simulations of a DNA dodecamer by Piana (Piana 2005).

The periodicity of $31 \AA$ observed in the diffraction patterns of stretched DNA films which had been crosslinked by brushing with formaldehyde solution (see Fig. 6) is very close to the pitch of C-DNA (Marvin et al. 1961; Saenger 1988; Fuller et al. 2004). The C conformation was indeed observed earlier in stretched DNA fibres, albeit at much lower Na content and humidity (Fornells et al. 1983). Other than this, however, there is little to support the presence of C-DNA in our samples: the expected second layer line of C-DNA, and the Raman band at around 870$884 \mathrm{~cm}^{-1}$ characteristic for C-DNA (Erfurth et al. 1972; Goodwin and Brahms 1978) are absent.

The reflection at $14.3 \AA$ shown in Fig. 6 is close to the second layer line reflection of A-DNA. However, the first layer line reflection at $28.6 \AA$ of A-DNA is not observed, nor its marked sixth, seventh and eighth layer line reflections.

Relating the stretch-induced structural changes observed in the present study to the ones predicted by molecular simulation is not straightforward, as the local forces acting on DNA within films are not well known. Although the strains in our experiments cover the entire range studied so far experimentally and theoretically with single molecules, a force plateau resembling the one observed in singlemolecule experiments and simulations cannot be seen in force-extension curves even from strongly cross-linked, elastomeric films (see Fig. 3b,c). This is probably due to disorder and entanglements within the film and may arise from film annealing after spinning. 


\section{Conclusions}

Wet-spun DNA films that were cross-linked and stretched at high humidity, and untreated films prepared with high spinning speed, show meridional X-ray diffraction spots corresponding to axial periodicities of 7.47 .8 and $8.2 \AA$ that are absent in all of the classical conformations normally observed for natural mixed sequence DNA (i.e. A, B, and $\mathrm{C}$ conformations). While the present data do not allow a definitive structural analysis to be carried out, they do demonstrate that major structural changes can occur in response to this type of sample treatment. Further development of sample preparation methods are in progress and may allow a clearer characterisation of this novel DNA conformation. Furthermore it is possible that improved data may be obtained through the use of repetitive sequence DNA polymers such as poly[d(GC)] - poly[d(GC)] and poly[d(AT)] - poly[d(AT)], whose conformational polymorphism is known to be different from that of random sequence DNA (Fuller et al. 2004).

Acknowledgment This work was funded by the Deutsche For schungsgemeinschaft. We thank A. Rupprecht and H. Grimm for teaching us the wet spinning method, P. Seige, A. Schiefner, K. Diederichs and $\mathrm{W}$. Welte for help with $\mathrm{X}$ ray diffraction, U. Lucchesi and H. Schneider for help with Raman measurements, I. Rouzina and R. Lavery for helpful discussions, I. Seuffert for help with DNA preparation and T. Meergans for biochemical advice.

\section{References}

Ahsan A, Rudnick J, Bruinsma R (1998) Elasticity theory of the B DNA to S DNA transition. Biophys J 74:132 137

Arnott S, Chandrasekaran R, Birdsall DL, Leslie AGW, Ratliff RL (1980) Left handed DNA helices. Nature 283:743 745

Beland FA, Fullerton NF, Heflich RH (1984) Rapid isolation, hydrolysis and chromatography of formaldehyde modified DNA. J Chromatogr (Biomed Appl) 308:121 131

Brodolin K (2000) Protein DNA crosslinking with formaldehyde in vitro. In: Travers A, Buckle M (eds) DNA protein interactions: a practical approach. Oxford University Press, Oxford, pp 141 150

Chaw YFM, Crane LE, Lange P, Shapiro R (1980) Isolation and identification of cross links from formaldehyde treated nucleic acids. Biochemistry 19:5525 5531

Cluzel P, Lebrun A, Heller C, Lavery R, Viovy J L, Chatenay D, Caron F (1996) DNA: an extensible molecule. Science 271:792 794

Cocco S, Yan J, Léger JF, Chatenay D, Marko JF (2004) Overstret ching and force driven strand separation of double helix DNA. Phys Rev E 70:011910 118

Collins CJ, Guild WR (1968) Irreversible effects of formaldehyde on DNA. Biochim Biophys Acta 157:107 113

Erfurth SC, Kiser EJ, Peticolas WL (1972) Determination of the backbone structure of nucleic acids and nucleic acid oligomers by laser Raman scattering. Proc Natl Acad Sci USA 69:938 941

Erfurth SC, Peticolas WL (1975) Melting and premelting phenom enon in DNA by laser Raman scattering. Biopolymers 14:247 264
Fornells M, Campos JL, Subirana JA (1983) Changes of conformation of DNA produced by mechanical forces. J Mol Biol 166:249 252

Franklin RE, Gosling RG (1953) The structure of sodium thymo nucleate fibres. I. The influence of water content. Acta Cryst 6:673 677

Freifelder D, Davison PF (1963) Physicochemical studies on the reaction between formaldehyde and DNA. Biophys J 3:49 63

Fuller W, Forsyth T, Mahendrasingam A (2004) Water DNA interactions as studied by $\mathrm{X}$ ray and neutron fibre diffraction. Phil Trans R Soc Lond B 359:1237 1248

Goodwin DC, Brahms J (1978) Form of DNA and the nature of interactions with proteins in chromatin. Nucleic Acids Res 5:835 850

Greenall RJ, Nave C, Fuller W (2001) X ray diffraction from DNA fibres under tension. J Mol Biol 305:669 672

Harris SA, Sands ZA, Laughton CA (2005) Molecular dynamics simulations of duplex stretching reveal the importance of entropy in determining the biomechanical properties of DNA. Biophys J 88:1684 1691

Huang H, Hopkins PB (1993) DNA interstrand cross linking by formaldehyde: nucleotide sequence preference and covalent structure of the predominant cross link formed in synthetic oligonucleotides. J Am Chem Soc 115:9402 9408

Huang H, Solomon MS, Hopkins PB (1992) Formaldehyde prefer entially interstrand cross links duplex DNA through deoxyadenosine residues at the sequence $5^{\prime} \mathrm{d}(\mathrm{AT})$. J Am Chem Soc 114:9240 9241

Huang Z, McWilliams A, Lam S, English J, McLean DI, Lui H, Zeng $\mathrm{H}$ (2003) Effect of formalin fixation on the near infrared Raman spectroscopy of normal and cancerous human bronchial tissues. Int J Oncol 23:649 655

Kosikov KM, Gorin AA, Zhurkin VB, Olson WK (1999) DNA stretching and compression: large scale simulations of double helical structures. J Mol Biol 289:1301 1326

Lai P Y, Zhou Z (2003) Stretching a double stranded DNA: nature of the B form to the $\mathrm{S}$ form transition. J Chem Phys 118:11189 11199

Lavery R, Lebrun A, Allemand JF, Bensimon D, Croquette V (2002) Structure and mechanics of single biomolecules: experiment and simulation. J Phys Cond Matter 14:R383 R414

Lebrun A, Lavery R (1996) Modelling extreme stretching of DNA. Nucleic Acids Res 24:2260 2267

Lee SA, Grimm H, Pohle W, Scheiding W, van Dam L, Song Z, Levitt MH, Korolev N, Szabó A, Rupprecht A (2000) NaDNA bipyridyl (ethylenediamine)platinum (II) complex: structure in oriented wet spun films and fibers. Phys Rev E 62:7044 7058

Lehner R, Koota J, Maret G, Gisler T (2006) Segment distributions of end tethered polymers in a good solvent. Phys Rev Lett 96:107801 14

MacKerell AD, Lee GU (1999) Structure, force, and energy of a double stranded DNA oligonucleotide under tensile loads. Eur Biophys J 28:415 426

Marko JF (1998) DNA under high tension: overstretching, under twisting, and relaxation dynamics. Phys Rev E 57:2134 2149

Marvin DA, Spencer M, Wilkins MHF, Hamilton LD (1961) The molecular configuration of deoxyribonucleic acid. III. $\mathrm{X}$ ray diffraction study of the $\mathrm{C}$ form of the lithium salt. J Mol Biol 3:547 565

Peticolas WL, Kubasek WL, Thomas GA, Tsuboi M (1987) Nucleic acids. In: Spiro TG (ed) Biological Applications of Raman Spectroscopy, vol 1. Wiley, New York, pp 81133

Piana S (2005) Structure and energy of a DNA dodecamer under tensile load. Nucleic Acids Res 33:7029 7038

Rief M, Clausen Schaumann H, Gaub HE (1999) Sequence depen dent mechanics of single DNA molecules. Nat Struct Biol $6: 346349$ 
Rouzina I, Bloomfield VA (2001) Force induced melting of the DNA double helix. 1. Thermodynamic analysis. Biophys J 80:882 893

Rouzina I, Bloomfield VA (2001) Force induced melting of the DNA double helix. 2. Effect of solution conditions. Biophys J 80:894 900

Rupprecht A (1963) Preparation of oriented DNA in large amounts. Biochem. Biophys Res Commun 12:163

Rupprecht A (1966) Preparation of oriented DNA by wet spinning. Acta Chem Scand 20:494

Rupprecht A (1970) Mechanochemical study of wet spun lithium DNA fibers. Biopolymers 9:825 842

Rupprecht A, Forslind B (1970) Variation of electrolyte content in wet spun lithium and sodium DNA. Biochim Biophys Acta 204:304

Saenger W (1988) Principles of nucleic acid structure. Springer, New York

Sarkar A, Léger JF, Chatenay D, Marko JF (2001) Structural transitions in DNA driven by external force and torque. Phys Rev E 63:051903

Smith SB, Cui Y, Bustamante C (1996) Overstretching B DNA: the elastic response of individual double stranded and single stranded DNA molecules. Science 271:795 799
Smith SB, Finzi L, Bustamante C (1992) Direct mechanical measurements of the elasticity of single DNA molecules by using magnetic beads. Science 258:1122 1126

Southern EM (1979) Measurement of DNA length by gel electro phoresis. Anal Biochem 100:319 323

Storm C, Nelson P (2003) Theory of high force DNA stretching and overstretching. Phys Rev E 67:51906 112

Wilkins MHF (1961) The molecular structure of DNA. J Chim Phys 58:891 898

Wilkins MHF, Gosling RG, Seeds WE (1951) Nucleic acid: an extensible molecule? Nature 167:759 760

Williams MC, Wenner JR, Rouzina I, Bloomfield VA (2001) Effect of $\mathrm{pH}$ on the overstretching transition of double stranded DNA: Evidence of force induced DNA melting. Biophys J 80:874 881

Williams MC, Wenner JR, Rouzina I, Bloomfield VA (2001) Entropy and heat capacity of DNA melting from temperature dependence of single molecule stretching. Biophys J 80:1932 1939 